Research Paper

\title{
Retrospective Evaluation of Standard Diagnostic Procedures in Identification of the Causes of New-Onset Syndrome of inappropriate Antidiuresis
}

\author{
Chih-Yang Hsu¹, Chieh-Liang Chen¹,2, Wei-Chieh Huang1, Po-Tsang Lee1, 2, Hua-Chang Fang1,2, Kang-Ju \\ $\mathrm{Chou}^{1,2 \bowtie}$
}

1. Division of Nephrology, Department of Internal Medicine, Kaohsiung Veterans General Hospital, Kaohsiung, Taiwan

2. National Yang-Ming University, School of Medicine, Taipei, Taiwan

$\square$ Corresponding author: Kang-Ju Chou, M.D., Division of Nephrology, Department of Internal Medicine, Kaohsiung Veterans General Hospital. \#386, Ta-Chung 1st Road, Kaohsiung 813, Taiwan. Tel: +886-7-342-2121 ext. 2043; Fax: +886-7-345-5412; E-mail: kjchou@vghks.gov.tw

(c) Ivyspring International Publisher. This is an open-access article distributed under the terms of the Creative Commons License (http://creativecommons.org/ licenses/by-nc-nd/3.0/). Reproduction is permitted for personal, noncommercial use, provided that the article is in whole, unmodified, and properly cited.

Received: 2013.03.2I; Accepted: 2013.12.24; Published: 2014.01.10

\begin{abstract}
Background: Many diagnostic procedures are conducted in patients with syndrome of inappropriate antidiuresis (SIAD). However, the contribution in identification of the cause of SIAD remains unknown.

Methods: The study was conducted at Kaohsiung Veterans General Hospital in southern Taiwan. From January 2000 to December 2009, medical records of 439 adult patients hospitalized for new-onset SIAD at a single center were retrospectively collected. All diagnostic procedures during hospitalization were divided into four groups: chest/lung, central nervous system, abdomen, and bone marrow to evaluate their positive rate leading to the cause of SIAD. Factors associated with "procedures leading to the cause" were also analyzed to improve efficacy of survey.

Results: Cause of SIAD was identified in 267 (60.8\%). Of them, I 50 were pulmonary disorders, 44 were drugs, 37 were central nervous system disorders, 32 were malignancy and 4 were post-surgery. Survey for chest/lung, central nervous system, abdomen, and bone marrow were performed in $96.6 \%, 29.2 \%, 38.0 \%$ and $3.6 \%$ of patients, respectively; positive findings leading to the cause of SIAD were $39.6 \%, 12.5 \%, 5.3 \%$ and $6.3 \%$, respectively. Among the diagnostic procedures, chest $x$-ray $(424 / 439,96.6 \%)$ was most frequently performed with the highest identification rate of $34.7 \%$ ( 147 cases). Major significant independent factors that associated with "procedure leading to a cause" were: absence of SIAD-associated drug history, presence of fever/chills, and presence of respiratory symptoms. Cause of SIAD became evident later during the follow-up period in 10 of 172 (5.8\%) patients who were initially thought to be cause-unknown. Malignancy was the cause for 5 cases and pulmonary tuberculosis was for the other five. Eight of these causes became evident within one year after the diagnosis of SIAD.

Conclusions: SIAD with unidentified causes were prevalent. Current diagnostic procedures remain not satisfying in determining the cause of SIAD, but chest radiograph did demonstrate higher diagnostic rate, especially in patients presented with fever, chills, respiratory symptoms, and without SIAD-associated drug history. Patients with unidentified cause should be followed for at least one year when most hidden causes (e.g. malignancy and tuberculosis) become obvious.
\end{abstract}

Key words: Diagnostic procedures; Hyponatremia; Syndrome of inappropriate antidiuresis (SIAD); Syndrome of inappropriate antidiuretic hormone (SIADH) 


\section{Introduction}

Syndrome of inappropriate antidiuresis (SIAD) is the most common cause of euvolemic hyponatremia. It can be caused by many clinical conditions, including malignancy, pulmonary disorders, central nervous system disorders, infections, and drugs [1]. To resolve SIAD, clinicians should make an effort to explore its cause and then treat the patient accordingly and appropriately. Previous reports have disclosed that patients might receive a series of diagnostic procedures such as abdominal ultrasound (US), computerized tomography (CT), magnetic resonance imaging (MR), bronchoscopy, and electroencephalography (EEG) if their causes were not immediately apparent [2-3]. Extensive survey seems reasonable, but is costly and time-consuming. One report estimated the annual costs of hyponatremia in the United States, showing that chest $\mathrm{x}$-ray, CT and MR were respectively performed in $100 \%, 25 \%$ and $25 \%$ of the patients with SIAD [4]. These examinations resulted in an increased length of stay and additional costs, but their true diagnostic value remains unknown.

To the best of our knowledge, the only study that evaluated the diagnostic procedures in exploring the causes of SIAD was from Hirshberg et al. [5]. They evaluated 50 elderly patients and found that $60 \%$ were idiopathic. Further image or invasive diagnostic studies in exploring the cause of SIAD were often futile. It concluded that simple diagnostic procedures including history review, physical examination and chest film are enough to make a treatable diagnosis in elderly patients. Although Hirshberg's suggestion was questioned owing to the lack of outcome data to support its accuracy [6], it offered a good direction to conduct procedures. In the era that chest film had almost become the routine procedure in patients with SIAD [4], it remains unknown whether the cause of SIAD were adequately identified. In addition, it had been reported that some causes of SIAD are initially undetectable and become evident later [7-9], developing a problem about what is the optimal policy to manage patients with unidentified cause. Therefore, we conducted the study and tried to answer these problems.

\section{Methods}

All adult patients being hospitalized for SIAD in our institution, an urban-based veteran hospital in southern Taiwan, between January 2000 and December 2009 were enrolled in the study and data were collected and reviewed retrospectively. Based on International Classification of Diseases, Ninth Revision, Clinical Modification (ICD-9-CM), all patients included were diagnosed with SIAD (diagnosis code:
253.6). Patients were included if they aged $>15$ years old and met the diagnostic criteria described by Bartter and Schwartz [10]: (1) hypo-osmolar hyponatremia; (2) urine osmolality $>100 \mathrm{mOsmol} / \mathrm{kg}$; (3) urine sodium concentration $>40 \mathrm{mEq} / \mathrm{L}$; (4) euvolemic state; (5) normal renal, thyroid and adrenal function. Thyroid function was considered normal if the level of serum high-sensitive TSH and free T4 were within a normal range [11]. Normal adrenal function was defined as a morning serum cortisol concentration $>15 \mathrm{ug} / \mathrm{dL}$ [12].

Clinical data, including demographic information, clinical and laboratory findings and medical history were obtained from comprehensive chart review. Several clinical terms were defined. Positive drug history meant that patients were recently exposed to SIAD-associated drugs, including chlorpropramide, selective serotonin-reuptake inhibitors (SSRIs), tricyclic antidepressants, clofibrate, carbamazepine, vincristine, nicotine, narcotics, antipsychotic drugs, ifosfamide, cyclophosphamide, nonsteroidal antiinflammatory drugs (NSAIDs), vasopressin, oxytoxin, desmopressin, etc [1]. Diabetes was defined by ICD-9-CM diagnosis codes of 250.xx, 357.2, 362.0, and 366.41; hypertension was defined by codes of 401.xx to 405.xx. Respiratory symptoms included cough, dyspnea, chest pain, rhinorrhea, hemoptysis, wheezing or respiratory failure, etc. Focal neurological deficits included numbness, weakness, paralysis, paresis, paresthesia or convulsion of focal limbs; impairment of speech, vision or hearing; nystagmus, vertigo, unsteady gait, falls, etc. Non-focal neurological deficits included dizziness, headache, generalized weakness, dementia, delirium, depression, anxiety, memory impairment and decrease of conscious level, etc. Gastrointestinal symptoms included nausea, vomit, decreased appetite, hematemesis, melena, abdominal pain, weight loss, constipation, diarrhea, etc. Genitourinary symptoms included flank pain, pelvic pain, dysuria, hematuria, urgency, frequency, incontinence, abnormal menstruation, vaginal/urethral discharge, etc.

To determine the cause of SIAD, we used following criteria to enhance the accuracy of diagnosis. First, a condition was considered to be the possible cause of SIAD if it had been documented in previous report. In this study, only a limit set of common causes listed in Ellison's review article were considered [1]. Other possible causes such as nephrogenic syndrome of inappropriate antidiuresis (NSIAD) were not considered in this study. Second, the cause was considered to be identified if a treatable condition (such as drugs, treatable infections or removable tumors) resolved with a normalized serum sodium level ( $\geq 135 \mathrm{meq} / \mathrm{L}$ ); or an incurable condition (such as ma- 
lignancy in terminal stage or refractory respiratory failure) with sustained hyponatremia till the date of mortality or loss of follow-up. Third, the cause was considered as unknown if a patient had none of the cause listed in Ellison's review article; or had a paradoxical response of serum sodium level to treatment (e.g., serum sodium level was normalized before a disease was resolved, or sustained hyponatremia even if a disease is cured).

Because disorders that result in SIAD were commonly originate from chest/lung, central nervous system, gastrointestinal/genitourinary tract (abdomen) and bone marrow [1], we evaluated procedures below that were commonly used to make diagnosis for these systems. For instance, chest x-ray, US/CT of chest, sputum studies (including culture for microorganisms and cytology) and bronchoscopy were frequently used to evaluate for chest and lung. The CT/MR of brain, cerebrospinal fluid (CSF) analysis and EEG were used for central nervous system. The abdominal plain film (APF), US/CT/MR of abdomen, esophagogastroduodenoscopy (EGD), colorectal endoscopy (CRE), double-contrast barium enema (DCBE), urine studies (including culture for microorganisms and cytology), intravenous pyelography (IVP) and urological endoscopy (UE) were used for abdomen. Bone marrow study included culture for microorganisms, cytology and pathologic examination. Procedure leading to the cause was defined if its finding first disclosed an impression or diagnosis of the cause of SIAD. For example, if a lung mass was first found by chest $x$-ray and following sputum cytology revealed a malignancy, chest $x$-ray was the procedure that leading to the cause of SIAD.

Data were expressed as the mean values \pm standard deviation, or number (percentage). Categorical variables were compared using the chi-square test or Fisher's exact test when appropriate. Quantitative variables were compared using Student $t$-tests. To identify the patients who were associated with positive result of survey, stepwise multivariate logistic regression analysis was performed to determine the independent factors. Factors with a $P$ value of less than 0.05 were put in a regression mode for analysis. Two-sided tests of significance were used and the results were considered to be significant with a $P$ value of less than 0.05 . Statistical analyses were conducted using SPSS 17.0 (SPSS Inc., Chicago, Illinois, USA).

This study was approved by the Institutional Review Board of the Kaohsiung Veterans General Hospital (No. VGHKS12-CT2-01).

\section{Results}

A total of 787 episodes of SIAD developed in 720 patients. Among them, 104 patients were excluded because 4 were aged under 15, 36 did not fit Bartter and Schwartz laboratory criteria, and 64 had no data of thyroid or adrenal function. Another 177 patients were also excluded because they had chronic SIAD or chronic hyponatremia before hospitalization. At last, 439 patients with new-onset of SIAD were included for further analyses (Table 1). Average age of the population sample was $75.0 \pm 11.4$ years. Among them, 299 patients $(68.1 \%)$ were male. When analyzing preexisting medical conditions of the patients, 71 $(16.2 \%)$ patients had positive drug history, 104 (23.7\%) had diabetes, and 196 (44.6\%) had hypertension. The clinical presentations on admission were: fever/chills in 129 (29.4\%), respiratory symptoms in 123 (28.0\%), focal neurological symptoms in $74(16.9 \%)$, non-focal neurological symptoms in 297 (67.7\%), gastrointestinal symptoms in 95(21.6\%), and genitourinary symptoms in $21(4.8 \%)$. Laboratory findings were listed in Table 1.

Table I. Patient characteristics

\begin{tabular}{ll}
\hline Characteristic & $\begin{array}{l}\text { Total } \\
(\mathrm{n}=439)\end{array}$ \\
\hline Age (yrs) & $75.0 \pm 11.4$ \\
Sex (male) & $299(68.1)$ \\
Positive drug history & $71(16.2)$ \\
Diabetes & $104(23.7)$ \\
Hypertension & $196(44.6)$ \\
Symptoms & \\
Fever/chills & $129(29.4)$ \\
Respiratory & $123(28.0)$ \\
Neurological, focal & $74(16.9)$ \\
Neurological, non-focal & $297(67.7)$ \\
Gastrointestinal & $95(21.6)$ \\
Genitourinary & $21(4.8)$ \\
Laboratory findings & \\
Sodium, serum (meq/L) & $124.3 \pm 4.8$ \\
BUN, serum (mg/dL) & $8.9 \pm 3.1$ \\
Creatinine, serum (mg/dL) & $0.8 \pm 0.2$ \\
Uric acid, serum (mg/dL) & $3.5 \pm 1.6$ \\
Sodium, urine (meq/L) & $79.9 \pm 32.2$ \\
Osmolality, urine (mmol/L) & $399.3 \pm 125.4$ \\
Cortisol, serum (ug/dL) & $25.7 \pm 8.7$ \\
Free thyroxine (ng/dL) & $1.3 \pm 0.3$ \\
HS-TSH (uIU/mL) & $1.5 \pm 1.0$ \\
\hline
\end{tabular}

Data are expressed as mean value $\pm \mathrm{SD}$, or no. (\%). Abbreviations: BUN: blood urea nitrogen; HS-TSH: high-sensitivity thyroid-stimulating hormone.

The causes of SIAD were identified in 267 $(60.8 \%)$ patients (Table 2). Of them, 150 were pulmonary disorders, 44 were drugs, 37 were central nervous system disorders, 32 were malignancy and 4 were post-surgery. Serum sodium level became normalized after management for 233 (53.1\%) patients. Among these patients, serum sodium level would become 
completely normal for those whose causes of SIAD were drug related reactions and post-surgery related reactions. Other than those cases, patients with pulmonary diseases were the most likely to become normonatremic ( 89 of 150,59.3\%), followed by central nervous system disorders (19 of $37,51.4 \%)$, unidentified cause (69 of 172, 40.1\%) and malignancy (8 of 32, $25.0 \%)$.

Table 2. The cause of SIAD

\begin{tabular}{|c|c|c|}
\hline Cause & $\begin{array}{l}\text { Number }(\%) \text { of } \\
\text { Patients (Total }=439)\end{array}$ & $\begin{array}{l}\text { Hyponatremia } \\
\text { resolved }\end{array}$ \\
\hline Cause-unidentified & $172(39.2)$ & 69 \\
\hline Cause-identified & $267(60.8)$ & 164 \\
\hline Pulmonary disorders & $150(34.2)$ & 89 \\
\hline Pneumonia & 96 & 65 \\
\hline Tuberculosis & 43 & 20 \\
\hline $\begin{array}{l}\text { Respiratory failure with posi- } \\
\text { tive-pressure breathing }\end{array}$ & 7 & 3 \\
\hline Pulmonary abscess/empyema & 4 & 1 \\
\hline Drugs & $44(10.0)$ & 44 \\
\hline SSRIs & 24 & \\
\hline NSAIDs & 5 & \\
\hline Carbamazepine & 4 & \\
\hline TCAs & 4 & \\
\hline Anti-psychotic agents & 2 & \\
\hline Desmopressin & 2 & \\
\hline Cyclophosphamide & 2 & \\
\hline Ifosfamide & 1 & \\
\hline Central nervous disorders & $37(8.4)$ & 19 \\
\hline Ischemic stroke & 14 & 6 \\
\hline Intracranial hemorrhage & 10 & 6 \\
\hline Meningitis & 5 & 2 \\
\hline Neoplasm, benign & 3 & 3 \\
\hline Hydrocephalus & 2 & 1 \\
\hline Brain abscess & 2 & 1 \\
\hline Multiple sclerosis & 1 & 0 \\
\hline Malignant diseases & $32(7.3)$ & 8 \\
\hline Lung cancer & 18 & 2 \\
\hline Gastrointestinal cancer & 5 & 2 \\
\hline Malignant lymphoma & 3 & 1 \\
\hline Genitourinary cancer & 3 & 3 \\
\hline Malignant brain tumor & 2 & 0 \\
\hline Head and neck cancer & 1 & 0 \\
\hline Post surgery & $4(0.9)$ & 4 \\
\hline
\end{tabular}

A total of 1,184 procedures were done for 434 of 439 (98.9\%) patients. In 267 cause-identified patients, $194(72.7 \%)$ were identified by these procedures (Table 3). There were 424 (96.6\%) patients receiving procedures for chest and lung and the cause of SIAD was then identified in 168 (39.6\%). Chest x-ray (424 of 439, $96.6 \%$ ) was the most frequently performed procedure, followed by sputum study $(256,58.3 \%)$, CT of chest $(42,9.6 \%)$, bronchoscopy $(22,5.0 \%)$ and US of chest (3, $0.7 \%)$. Among these studies, chest $x$-ray had the highest identification rate (147 of $424,34.7 \%$ ) as a procedure leading to the cause of SIAD, followed by CT of chest (4 of $42,9.5 \%$ ) and sputum study (17 of 256, 6.6\%). Procedures for central nervous system were offered to 128 (29.2\%) patients, which identified the cause of SIAD for $16(12.5 \%)$ cases. CT of brain was the prominent procedure (117 of $439,26.7 \%)$, followed by MR of brain $(26,5.9 \%)$, CSF study $(16,3.6 \%)$ and EEG (1, 0.2\%). Of them, CSF study had the highest identification rate (5 of $16,31.3 \%$ ) as a procedure leading to the cause, followed by CT of brain (10/117, $8.5 \%$ ) and MRI of brain (1 of 26, 3.8\%). Procedures for abdomen were offered to 167 (38\%) patients, which identified the cause of SIAD for $9(5.3 \%)$. APF was the prominent procedure (105 of 439, 23.9\%), followed by US of abdomen (70, 15.9\%), EGD (29,6.6\%), CT of abdomen $(25,5.7 \%)$, urine studies $(22,5.0 \%)$, CRE (6, $1.4 \%), \operatorname{DCBE}(3,0.7 \%)$ and MR of abdomen (1, 0.2\%). No patients were found to receive IVP or UE. Of them, US of abdomen (6 of 70,8.6\%) had a higher rate as a procedure leading to the cause, followed by CT of abdomen ( 2 of $25,8.0 \%)$ and EGD (1 of $29,3.4 \%)$. Bone marrow study was performed in 16 of 439 (3.6\%) patients and as a procedure leading to the cause in 1 (6.3\%). US of chest, bronchoscopy, EEG, APF, MR of abdomen, CRE, DCBE and urine studies were not noted as a procedure leading to the cause.

Patients were further divided into two groups: those with procedure leading to a cause of SIAD $(n=194)$ and those without $(n=245)$. Comparison of clinical characteristics of these two groups of patients was then done. In univariate analysis, factors that associated with procedure leading to a cause of SIAD included younger age, male sex, absence of drug history, absence of hypertension, presence of fever/chills, and presence of respiratory symptoms (Table 4). Further results of multivariate logistic regression analysis showed that procedure leading to the cause of SIAD was borderline significantly associated with age (OR:1.023 per one year younger, 95\% CI: 1.003-1.045, $p=0.041$ ), but was much significantly associated with presence of respiratory symptoms (OR:12.417; 95\% CI: 6.989-22.060, $p<0.001$ ), absence of SIAD-associated drug history (OR:4.669; 95\% CI: 2.140-10.184, $p<0.001$ ), and presence of fever/chills (OR:2.950, 95\% CI: 1.785-4.875, $p<0.001$ ). (Table 5).

During the mean follow-up period of 36.8 months, the cause of SIAD became evident later in 10 of $172(5.8 \%)$ cause-unidentified patients (Table 6). Among them, pulmonary tuberculosis was the cause for 5 cases and malignancy was for the other five. For the five patients with pulmonary tuberculosis, the initial diagnosis was bacterial pneumonia for three (patient 1-3) and fever of unknown origin for the other two (patient 4, 5). Mycobacterium tuberculosis was iso- 
lated later from sputum for two and gastric juice for three patients. Plasma sodium level was normalized in all patients after anti-tuberculosis therapy. The five patients with malignancy were diagnosed with small cell lung cancer, renal cell carcinoma, gastric adenocarcinoma, colon adenocarcinoma, and malignant lymphoma, respectively. Plasma sodium level was partially improved after chemotherapy was offered for patient 6 . Hyponatremia was completely resolved after removal of tumor in patient 7. Patient 8 to 10 received hospice care. Eight of these causes became evident within one year after the diagnosis of SIAD.

Table 3. The diagnostic procedures and the positive rate in exploring the causes of SIAD

\begin{tabular}{|c|c|c|c|c|c|}
\hline Site of survey & $\begin{array}{l}\text { Patient received pro- } \\
\text { cedures }\end{array}$ & Cause identified & $\begin{array}{l}\text { Diagnostic proce- } \\
\text { dures }\end{array}$ & Procedure performed & $\begin{array}{l}\text { Procedure leading to } \\
\text { the cause }\end{array}$ \\
\hline \multirow[t]{5}{*}{ Chest and lung } & \multirow[t]{5}{*}{$424 / 439(96.6)$} & \multirow[t]{5}{*}{$168 / 424(39.6)$} & Chest x-ray & $424 / 439(96.6)$ & $147 / 424(34.7)$ \\
\hline & & & US of chest & $3 / 439(0.7)$ & $0 / 3(0)$ \\
\hline & & & $\mathrm{CT}$ of chest & $42 / 439(9.6)$ & $4 / 42(9.5)$ \\
\hline & & & Sputum studies & $256 / 439(58.3)$ & $17 / 256(6.6)$ \\
\hline & & & Bronchoscopy & $22 / 439(5.0)$ & $0 / 22(0)$ \\
\hline \multirow{4}{*}{$\begin{array}{l}\text { Central nervous sys- } \\
\text { tems }\end{array}$} & \multirow[t]{4}{*}{$128 / 439(29.2)$} & \multirow[t]{4}{*}{$16 / 128(12.5)$} & CT of brain & $117 / 439(26.7)$ & $10 / 117(8.5)$ \\
\hline & & & MR of brain & $26 / 439(5.9)$ & $1 / 26(3.8)$ \\
\hline & & & CSF study & $16 / 439(3.6)$ & $5 / 16(31.3)$ \\
\hline & & & EEG & $1 / 439(0.2)$ & $0 / 1(0)$ \\
\hline \multirow[t]{8}{*}{ Abdomen } & \multirow[t]{8}{*}{$167 / 439(38.0)$} & \multirow[t]{8}{*}{$9 / 167(5.3)$} & $\mathrm{APF}$ & $105 / 439(23.9)$ & $0 / 105(0)$ \\
\hline & & & US of abdomen & $70 / 439(15.9)$ & $6 / 70(8.6)$ \\
\hline & & & CT of abdomen & $25 / 439(5.7)$ & $2 / 25(8.0)$ \\
\hline & & & MR of abdomen & $1 / 439(0.2)$ & $0 / 1(0)$ \\
\hline & & & EGD & $29 / 439(6.6)$ & $1 / 29(3.4)$ \\
\hline & & & CRE & $6 / 439(1.4)$ & $0 / 6(0)$ \\
\hline & & & DCBE & $3 / 439(0.7)$ & $0 / 3(0)$ \\
\hline & & & Urine studies & $22 / 439(5.0)$ & $0 / 22(0)$ \\
\hline Bone marrow & $16 / 439$ (3.6) & 1/16(6.3) & BMS & $16 / 439(3.6)$ & $1 / 16(6.3)$ \\
\hline
\end{tabular}

Table 4. Differences of clinical characteristics between patients that procedure leading to a cause of SIAD and not

\begin{tabular}{|c|c|c|c|}
\hline \multirow[b]{2}{*}{ Characteristic } & \multicolumn{2}{|c|}{ Procedure leading to the cause of SIAD } & \multirow[b]{2}{*}{ P value } \\
\hline & $\begin{array}{l}\text { Yes } \\
(n=194)\end{array}$ & $\begin{array}{l}\text { No } \\
(\mathrm{n}=245)\end{array}$ & \\
\hline Age (yrs) & $73.7 \pm 11.6$ & $76.6 \pm 11.1$ & 0.009 \\
\hline Sex (male) & $146(75.3)$ & $153(62.4)$ & 0.004 \\
\hline Positive drug history & $11(5.7)$ & $60(24.5)$ & $<0.001$ \\
\hline Diabetes & $39(20.1)$ & $65(26.5)$ & 0.116 \\
\hline Hypertension & $73(37.6)$ & $123(50.2)$ & 0.008 \\
\hline \multicolumn{4}{|l|}{ Symptoms } \\
\hline Fever/chills & $84(43.3)$ & $45(18.4)$ & $<0.001$ \\
\hline Respiratory & $104(53.6)$ & $19(7.8)$ & $<0.001$ \\
\hline Neurological, focal & $30(15.5)$ & $44(18.0)$ & 0.488 \\
\hline Neurological, non-focal & $131(67.5)$ & $166(67.8)$ & 0.959 \\
\hline Gastrointestinal & $44(22.7)$ & $51(20.8)$ & 0.638 \\
\hline Genitourinary & $7(3.6)$ & $14(5.7)$ & 0.305 \\
\hline \multicolumn{4}{|l|}{ Laboratory findings } \\
\hline Sodium, serum (meq/L) & $124.5 \pm 4.7$ & $124.2 \pm 4.9$ & 0.556 \\
\hline BUN, serum (mg/dL) & $9.1 \pm 3.3$ & $8.7 \pm 3.0$ & 0.252 \\
\hline Creatinine, serum $(\mathrm{mg} / \mathrm{dL})$ & $0.8 \pm 0.2$ & $0.8 \pm 0.3$ & 0.131 \\
\hline Uric acid, serum (mg/dL) & $3.6 \pm 1.8$ & $3.4 \pm 1.5$ & 0.435 \\
\hline Sodium, urine (meq/L) & $82.0 \pm 35.7$ & $78.1 \pm 29.2$ & 0.334 \\
\hline Osmolality, urine (mmol/L) & $407.4 \pm 115.4$ & $394.4 \pm 132.1$ & 0.280 \\
\hline Cortisol, serum (ug/dL) & $26.9 \pm 9.4$ & $24.8 \pm 8.1$ & 0.074 \\
\hline Free thyroxine (ng/dL) & $1.2 \pm 0.3$ & $1.3 \pm 0.3$ & 0.144 \\
\hline HS-TSH (uIU/mL) & $1.4 \pm 0.8$ & $1.6 \pm 1.1$ & 0.136 \\
\hline
\end{tabular}

Data are expressed as mean value \pm SD, or no. (\%). Abbreviations: BUN: blood urea nitrogen; HS-TSH: high-sensitivity thyroid-stimulating hormone. 
Table 5. Independent factors that associated with surveys leading to the causes of SIAD

\begin{tabular}{llll}
\hline Variables & Odds ratio & $\begin{array}{l}\text { 95\% Confidence } \\
\text { interval }\end{array}$ & $P$ value \\
\hline Age ( per one year younger) & 1.023 & 1.001 to 1.045 & 0.041 \\
Respiratory symptoms & 12.417 & 6.989 to 22.060 & $<0.001$ \\
$\begin{array}{l}\text { Absence of SIAD-associated } \\
\text { drug history }\end{array}$ & 4.669 & 2.140 to 10.184 & $<0.001$ \\
\begin{tabular}{llll} 
Fever/chills & 2.950 & 1.785 to 4.875 & $<0.001$ \\
\hline Abbreviations: SIAD: syndrome of inappropriate antidiuresis
\end{tabular}
\end{tabular}

Table 6. Clinical profiles of ten patients whose cause of SIAD was identified later during the follow-up period

\begin{tabular}{llll}
\hline Patients & Age/sex & Cause of SIAD & $\begin{array}{l}\text { Time from SIAD to } \\
\text { cause identified } \\
\text { (months) }\end{array}$ \\
\hline 1 & $82 / \mathrm{F}$ & Pulmonary tuberculosis & 13.8 \\
2 & $77 / \mathrm{M}$ & Pulmonary tuberculosis & 12.6 \\
3 & $78 / \mathrm{M}$ & Pulmonary tuberculosis & 6.7 \\
4 & $80 / \mathrm{M}$ & Pulmonary tuberculosis & 2.4 \\
5 & $86 / \mathrm{M}$ & Pulmonary tuberculosis & 1.5 \\
6 & $73 / \mathrm{M}$ & Small cell lung cancer & 3.9 \\
7 & $69 / \mathrm{M}$ & Renal cell carcinoma & 10.5 \\
8 & $84 / \mathrm{M}$ & Malignant lymphoma & 2.3 \\
9 & $81 / \mathrm{M}$ & Gastric adenocarcinoma & 5.2 \\
10 & $78 / \mathrm{M}$ & Colon adenocarcinoma & 1.6 \\
\hline
\end{tabular}

\section{Discussion}

Our study showed that patients with new-onset SIAD received many diagnostic procedures during hospital stay. However, little of them were leading to the cause of SIAD. The results were similar to Hirshberg's report [5], presenting $92 \%, 58 \%$ and $16 \%$ of patients received chest x-ray, head CT and chest/abdomen CT, respectively, with a rate of positive findings leading to diagnosis only being $21 \%$, $10.3 \%$ and $25 \%$. In addition, unidentified causes of SIAD were noted for $39.2 \%$ in our study. It was also similar to early reports that revealed a percentage of unidentified causes of SIAD between $25.9 \%$ and $60 \%$ $[5,7,13]$. Different from early reports, we further demonstrated that most cause-unidentified patients did not develop a latent cause during the follow-up period. Whether these patients did have an idiopathic form of SIAD was unknown, but the high prevalence rate of unidentified causes reflected that current diagnostic procedures remain not satisfying in determining the cause of SIAD.

The most frequent procedure performed was chest x-ray, which was offered to $92 \%$ of Hirshberg's patients and $96.6 \%$ of ours. The concerns of lung cancer and the low-cost, less time-consumption characteristics of chest $\mathrm{x}$-ray might promote it to be excessively performed, but it did detect the most number of causes: $21 \%$ and $34.7 \%$ of positive rate in Hirshberg's and ours, respectively. The results also reflected that most causes of new-onset of SIAD were originated from chest and lung, leading to a relatively higher diagnostic rate of chest survey. In addition, chest infection seems more frequent than lung cancer in recent years. An earlier small case-series report from Kennedy et al. [14] described chest infections in 7 and lung cancer in 4 among 11 SIAD patients; Sunderam et al. [15] also presented 14 patients, describing chest infections in 5 and carcinoma in 4 . The proportion of chest infections became much higher than lung cancer in recent reports from Hirshberg et al. [5], who presented 50 patients, describing pneumonia in 9 and lung cancer in one. Our result also showed that pneumonia $(\mathrm{n}=96)$ and pulmonary tuberculosis $(n=43)$ were much more frequent than lung cancer $(n=18)$. The lower proportion of lung cancer might be due to the advance of imaging techniques and broad application of chest x-ray in health examination in developed countries, making lung cancer detected before the development of SIAD. Because chest x-ray is relatively low-cost and has long been suggested excellent to detect chest infection [16], it should be a reasonable diagnostic procedures in exploring the cause of SIAD.

Procedures for central nervous system were performed in $29.2 \%$ of our patients. Further analysis showed that procedures for central nervous system tended to be performed in patients presented with focal neurological symptoms (Odds ratio: 8.9, 95\% confident interval: 3.5-22.4, $\mathrm{p}<0.001)$. Because neurological presentations of hyponatremia are usually non-focal, it was reasonable to conduct a procedure to detect intracranial lesions in patients presenting focal neurological symptoms. CT of brain was the most common procedure for survey of central nervous system, but the diagnosed rate was not high. The low diagnostic rate might be caused by a part of our patients presented with ischemic stroke, which was occasionally undetectable by CT in early period. The MR of brain was less conducted, which would also possibly lead to low diagnostic rate. However, the diagnostic value of MR seemed not high because there was no life-threatening central nervous disorder developed during the follow-up period. For relative high cost and lower diagnostic rate, we do not suggest routine survey of central nervous disorders for patients with new onset of SIAD. Procedures for central nervous system may only be preserved for patients with obvious presentations indicating intracranial lesions (such as local neurological symptoms and signs).

Causes originated from abdomen or bone marrow were extremely few in our study. Similar findings were also noted in Hirshberg's report, in which zero 
cause was originated from these sites. Therefore, survey for these sites should only be considered in patients with obvious specific symptoms or after excluding pulmonary and central nervous disorders.

It had been suggested to simplify the diagnostic procedures in elderly patients because they seemed to have higher incidences of unidentified SIAD [5]. In our study, borderline significance was noted for age as an independent factor that associated with survey leading to the cause. As SIAD is prevalent in the elderly, we think that it is dangerous to establish an absolute cut-off point of age to simplify survey procedures.

We showed that some causes of SIAD were latent and initially undetectable. Causes that evaded detection had been described in sporadic case reports. Anpalahan [7] reported an 82-year-old male with an initial diagnosis of idiopathic SIAD. Metastatic bowel cancer became evident 12 weeks after the initial manifestation of hyponatremia. Martinez-Maldonado [8] identified a lung cancer in a 52-year-old woman who had been diagnosed as idiopathic SIAD by extensive survey 7 month ago. Furthermore, Renneboog et al [9] identified a nasal olfactory neuroblastoma in a 28-year-old woman with a history of idiopathic SIAD for 8 years. In our study, the latent causes were mainly pulmonary tuberculosis and malignancy. The prevalence rate of tuberculosis is high in Taiwan during the study periods [17]. In country less prevalent to pulmonary tuberculosis, malignancy should be first considered. Finally, it was also unclear about how long should be followed in cause-unknown SIAD patients. Except for Renneboog's patient, most reports and ours show that the duration for cause revealed was generally less than one year. As a result, this timeframe may be the minimum amount of time needed to follow such patients.

The main limitation of our study is its retrospective study design. Therefore, the methods to confirm euvolemia were not very precise. In our study, we found that the most common methods to confirm euvolemia were physical examination or poor response to correct hyponatremia by continuous $0.9 \%$ saline infusion. Water-load test was not performed in our patients due to concerns of worsening hyponatremia. In spite of the flaw, the average level of serum uric acid and serum BUN to creatinine ratio of our patients were $3.5 \pm 1.6 \mathrm{mg} / \mathrm{dL}$ and $11.7 \pm 6.1$, respectively. Both data indicated that most of our patients were not resulted from other cause of hyponatremia $[1,18,19]$. Further, because cosyntrosin is not available in Taiwan, the exclusion of adrenal insufficiency may not be complete. Few of our patients had data with regards to plasma arginine-vasopressin (AVP) and no one received vasopressin receptor an- tagonists, indicating the possibility that NSIAD may not be diagnosed. The syndrome fulfills the classic criteria of SIAD and is caused by a gain-of-functional mutation in the AVP receptor type 2, which was initially described in infants [20] and only recently reported in adult patients [21]. Finally, all included objects were hospitalized patients, indicating more severe manifestations, underlying disease and co-morbidity. Therefore, there was selection bias in the present study.

In conclusion, we found that SIAD with unidentified causes were prevalent. Current diagnostic procedures remain not satisfying in determining the cause of SIAD, but chest radiograph did demonstrate higher diagnostic rate, especially in patients presented with fever, chills, respiratory symptoms, and without SIAD-associated drug history. Patients with unidentified cause should be followed for at least one year when most hidden causes (e.g. malignancy and tuberculosis) become obvious.

\section{Competing Interests}

The authors have declared that no competing interest exists.

\section{References}

1. Ellison DH, Berl T. Clinical practice. The syndrome of inappropriate antidiuresis. N Engl J Med. 2007; 356: 2064-2072

2. Epstein FH, Levitin H, Glaser G, et al. Cerebral hyponatremia. N Engl J Med. 1961; 265: 513-518

3. Grumer HA, Derrybery W, Dubin A, et al. Idiopathic, episodic inappropriate secretion of antidiuretic hormone. Am J Med. 1962; 32: 954-963

4. Boscoe A, Paramore C, Verbalis JG. Cost of illness of hyponatremia in the United States. Cost Eff Resour Alloc. 2006; 4: 10

5. Hirshberg B, Ben-Yehuda A. The syndrome of inappropriate antidiuretic hormone secretion in the elderly. Am J Med. 1997; 103: 270-273

6. Sterns RH. The syndrome of inappropriate antidiuretic hormone secretion of unknown origin. Am J Kidney Dis. 1999; 33: 161-163

7. Anpalahan M. Chronic idiopathic hyponatremia in older people due to syndrome of inappropriate antidiuretic hormone secretion (SIADH) possibly related to aging. J Am Geriatr Soc. 2001; 49: 788-792

8. Martinez-Maldonado. Inappropriate antidiuretic hormone secretion of unknown origin. Kidney Int. 1980; 17: 554-567

9. Renneboog B, Decaux G. Idiopathic hyponatremia in a young patient: look at the sinus. Am J Med. 2008; 121: e5-6

10. Bartter FC, Schwartz WB. The syndrome of inappropriate secretion of antidiuretic hormone. Am J Med. 1967; 42: 790-806

11. Spencer CA. Clinical utility and cost-effectiveness of sensitive thyrotropin assays in ambulatory and hospitalized patients. Mayo Clin Proc. 1988; 63: 1214-1222

12. Grinspoon SK, Biller BM. Clinical review 62: Laboratory assessment of adrenal insufficiency. J Clin Endocrinol Metab. 1994; 79: 923-31

13. Miller M, Hecker MS, Friedlander DA, et al. Apparent idiopathic hyponatremia in an ambulatory geriatric population. J Am Geriatr Soc. 1996; 44: 404-8

14. Kennedy PG, Mitchell DM, Hoffbrand BI. Severe hyponatraemia in hospital inpatients. Br Med J. 1978; 2: 1251-1253

15. Sunderam SG, Mankikar GD. Hyponatremia in the elderly. Age Ageing. 1983; 12: 77-80

16. Bartlett JG, Dowell SF, Mandell LA, et al. Practice guidelines for the management of community-acquired pneumonia in adults. Infectious Diseases Society of America. Clin Infect Dis. 2000; 31: 347-382

17. Hsueh PR, Liu YC, So J, et al. Mycobacterium tuberculosis in Taiwan. J Inf. 2006; 52: 77-85

18. Beck LH. Hypouricemia in the syndrome of inappropriate secretion of antidiuretic hormone. N Engl J Med. 1979; 301: 528-530

19. Fenske W, Störk S, Koschker AC, et al. Value of fractional uric acid excretion in differential diagnosis of hyponatremic patients on diuretics. J Clin Endocrinol Metab. 2008; 93: 2991-2997

20. Feldman BJ, Rosenthal SM, Vargas GA, et al. Nephrogenic syndrome of inappropriate antidiuresis. N Engl J Med. 2005; 352: 1884-1890

21. Decaux G, Vandergheynst FR, Bouko Y, et al. Nephrogenic syndrome of inappropriate antidiuresis in adults: high phenotypic variability in men and women from a large pedigree. J Am Soc Nephrol. 2007; 18: 606-612 